\title{
TOTAL PHENOLIC COMPOUNDS AND TANNINS CONTENT OF BANCHA GREEN TEA (CAMELLIA SINENSIS) DEPENDING ON EXTRACTION CONDITIONS
}

\author{
Kaloyan Georgiev', Iliya Zhelev², Svetlana Georgieva² \\ ${ }^{1}$ Department of Preclinical and clinical sciences, Sector Pharmacology and Toxicology, \\ Faculty of Pharmacy, Medical University of Varna, ${ }^{2}$ Department of Pharmaceutical \\ technologies, Faculty of Pharmacy, Medical University of Varna
}

\begin{abstract}
The effect of different extraction conditions of prepared infusions on the content of bioactive compounds of Bancha green tea (Camellia sinensis L.) were investigated. The content of total phenols, tannins and methylxanthines were determined spectrophotometrically. The highest content of total phenolic compounds and tannis were determined on the $30^{\text {th }}$ minute of infusion $-7,71 \%$ and $4,49 \%$ respectively. The conditions with the most valuable (higher content of polyphenols) and the most healthy (lowest of tannins) combination is in the $10^{\text {th }}$ minute of extraction $-7.47 \%$ and $3,84 \%$. The tea also contained low percentages of methylxanthines $(0,66 \%)$ and caffeine $(0,09 \%)$.
\end{abstract}

Keywords: Bancha green tea, total phenols, flavonoids, methylxanthines, caffeine

\section{INTRODUCTION}

Green tea is a regularly consumed traditional drink in Japan and China and is characteristic to their culture. Although produced from the same plant, Camellia sinensis L., differences in the manufacturing process specify that green tea has a higher catechin content than black tea (1), which may contribute to its beneficial effects on cancer, cardiovascular diseases and other conditions (9). Tea contains large amounts of various phenolic compounds as flavonoids, phenolic acids, tannins and others. Based on data in literature, higher content of total phenols

Address for correspondence:

Kaloyan Georgiev

Department of Preclinical and clinical scinces

Faculty of Pharmacy

Medical University of Varna,

55 Marin Drinov Str., 9002 Varna, Bulgaria

e-mail: kalgeorgiev@hotmail.com

Received: February 21, 2014

Accepted: April 3, 2014 are connected with beneficial effects of the tea, while higher content of polymer phenolic - tannins is associated with adverse effects, mainly gastro-intestinal disorders (5). Another interesting group of phenolic compounds are the flavonoids. A class of tea flavanoids - catechins, include epicatechin (EC), epigallocaetchin (EGC), epicatechin-3-gallate (ECG), and epigallocatechin-3-gallate (EGCG) $(1,5)$ (Fig. 1). Other classes of compounds, important for their biological activity are methylxanthines. The main methylxanthine is caffeine, which has attracted much scientific and public attention during the past years. The caffeine content of tea leaves varies with tea type, but the normal range goes from 2-5\% (dry weight, w/w) together with small amounts of theobromine and theophyllin. Caffeine is chemically 1,3,7-trimethylxanthine $\left(\mathrm{C}_{8} \mathrm{H}_{10} \mathrm{~N}_{4} \mathrm{O}_{2}\right)$, which is an alkaloid found in tea, guarana, kola nuts, coffee, cocoa beans and other plants. Caffeine acts as a stimulant for the heart, respiratory and the central nervous system, and is a vasodilator as well as a diuretic (Fig. 1) (7). 


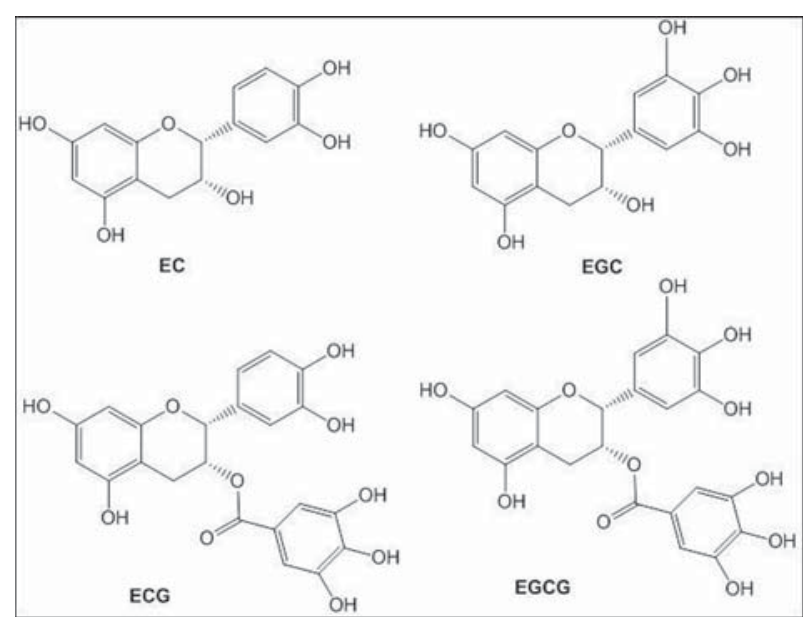

Fig. 1. Chemical structures of major green tea polyphenols: The polyphenols that exist in green tea are mainly (+)-epicatechin (EC), (-)-epigallocatechin (EGC), (-)-epicatechin gallate (ECG), and (-)-epigallocatechin gallate (EGCG). EGCG is the most powerful and the most abundant of the major catechins found in green tea

The main objectives of the present study is to determine the correlation between different extraction time and the content of total phenolic compounds and tannins in Bancha green tea as well as estimate the content of methylxanthines and especially the caffeine in it.

\section{MATERIAL AND METHODS}

\section{Plant materials and chemicals}

Whole Bancha green tea leaves (Camellia sinensis L.) were purchased from a local market. All chemicals used in the spectrophotometric methods were purchased from Sigma-Aldrich.

\section{Quantification of total phenols and tannins}

The determination of total phenols in the plant drugs was performed according to the European Pharmacopoeia 6 involving Folin-Chiocalteu reagent and pyrogallol as standard (4). The measurements were carried out using an S-22, UV/VIS spectrophotometer (Germany) at $760 \mathrm{~nm}$. caffeine

Quantification of total methylxanthines and

Accurately weighed amount of the drug $( \pm 0.0001 \mathrm{~g})$ were boiled in water for 15 minutes. The combined aqueous extracts were acidified with sulfuric acid and concentrated. The solution was extracted with chloroform in a separating funnel. Chloroform extract was washed with sodium hydroxide and then with water. After the evaporation of chloroform a mixture of methylxanthines was obtained. From this mixture the caffeine was prepared by sublimation then the percentage of caffeine in the plant substances was calculated (in w/w).

\section{Statistical analysis}

All experiments were done in triplicate and data in tables and figures represent mean values \pm standard deviation $(n=3)$. Results were evaluated for statistical significance using one-way ANOVA using SigmaPlot (Version 11.0). The confidence level for statistical significance was set at a probability value of 0.05 .

\section{RESULTS AND DISCUSSION}

Increasing interest in the health benefits of tea has led to the inclusion of tea extracts in dietary supplements and functional foods. Bancha green tea (third or fourth flush of green tea; that is, the late seasonal picking) (Fig. 2) is the one main types of green tea consumed in Japan, and is usually prepared by steeping the tea leaves in hot water (6).

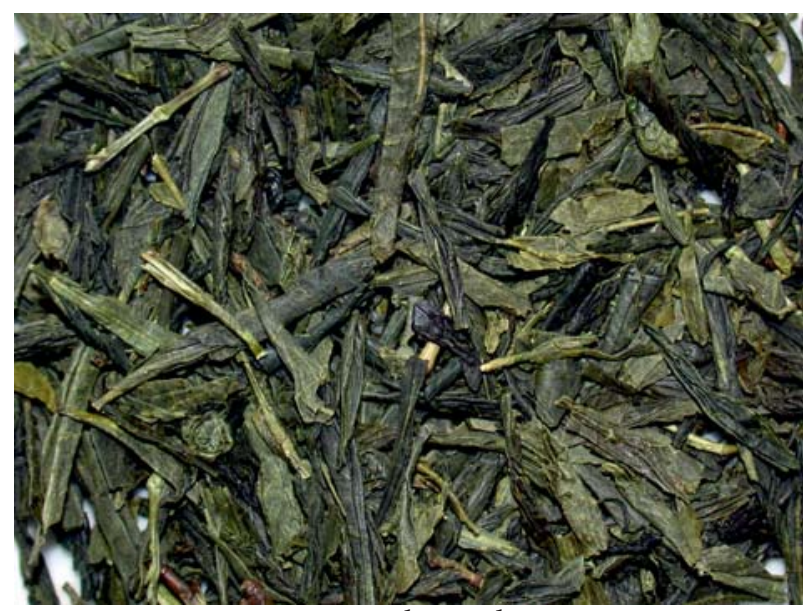

Fig. 2. Bancha tea leaves

In our study, we examined the impact of extraction time on total phenols and tannins content of Bancha green tea. Numerous extractions were conducted at different times between five and thirty minutes. Polyphenols content in these periods vary respectively between $6,99 \%(\mathrm{w} / \mathrm{w})$ to $7,71 \%(\mathrm{w} / \mathrm{w})$, and tannins between 3,65\% (w/w) to 4,49\% (w/w) (Fig. 3). The highest level of polyphenols $(7,71 \%(\mathrm{w} / \mathrm{w}))$ and tannins $(4,49 \%(\mathrm{w} / \mathrm{w}))$ were observed at the $30^{\text {th }} \mathrm{min}$ ute of extraction and lowest in the $5^{\text {th }}(6,99 \%(\mathrm{w} / \mathrm{w})$ and $3,65 \%(\mathrm{w} / \mathrm{w})$ for total polyphenols and tannins 
respectively). In the other investigated conditions the level of extracted polyphenols increased insignificantly from $7,47 \%(w / w)$, to $7,55 \%(w / w)$ while the rise of tannins was more elevated - 3,84\% (w/w) to $4,28 \%(\mathrm{w} / \mathrm{w})$. The conditions with the most valuable (higher content of polyphenols) and the most healthy (lowest of tannins) combination is at the $10^{\text {th }} \mathrm{min}$ ute of extraction where the content of total polyphenols was 7,47\% (w/w) and tannins only 3,84\% (w/w). Similar data for total polyphenols content were established in our previously study (8) with other types of tea (at the $30^{\text {th }}$ minute) - black and green tea, where phenolic compounds were 7,32\% (w/w) and 5,97\% $(\mathrm{w} / \mathrm{w})$ respectively. While the content of tannins is mainly associated with unpleasant taste - astringent feeling inside of your mouth and no beneficial systemic effects, the higher content of polyphenols provide for antioxidant, anti-inflammatory and cancer protective activity (Fig. 3) (10).

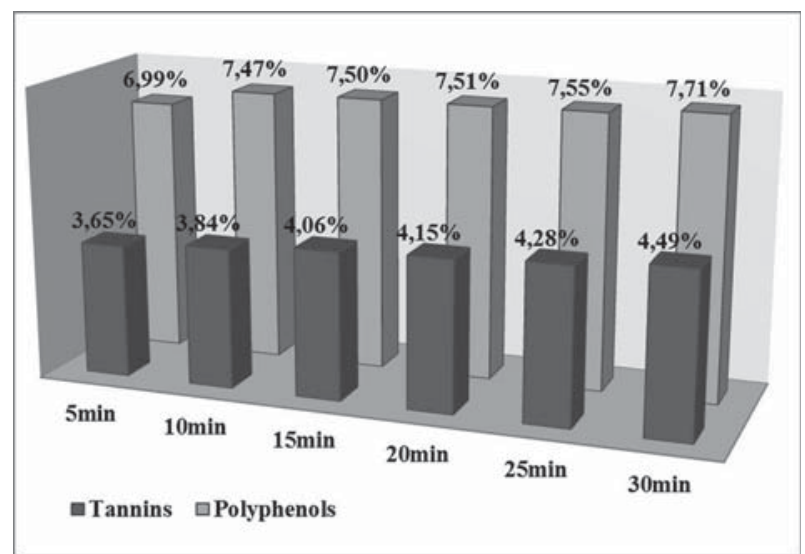

Fig. 3. Content $(\%, w / w)$ of polyphenols and tannins in Bancha tea leaves depending on extraction time (min)

Methylxanthines, in particular caffeine, is the world's most popular drug and is found in many beverages including tea. Although caffeine is commonly ingested to enhance alertness and improve performance, its use should be avoided by pregnant women, children, and people with cardiovascular diseases and anxiety disorders (3). In a further study, we have determined the content of total methylxanthines and caffeine. The results have shown that in Bancha tea total methylxanthines are $0,66 \%(\mathrm{w} / \mathrm{w})$, and caffeine is only 0,09\% (w/w) (Fig. 4).

In the above mentioned study (8), the content of total methylxanthines and caffeine in black and

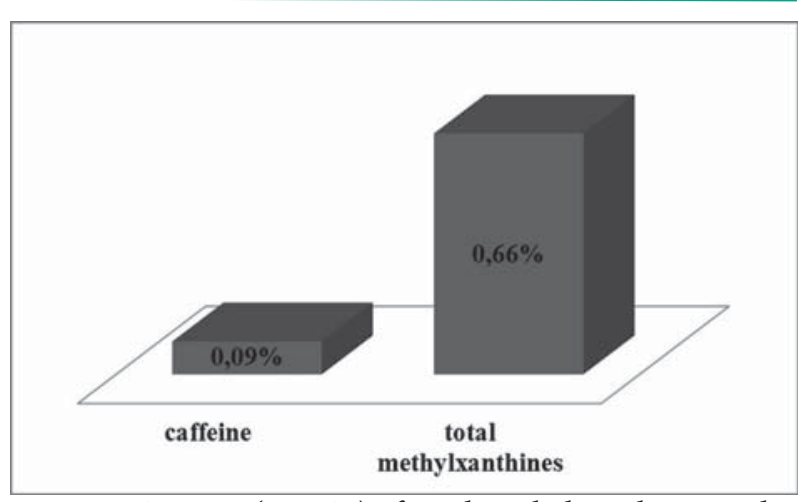

Fig. 4. Content $(\%, w / w)$ of total methylxanthines and caffeine in Bancha tea leaves

green tea made in the same extraction, conditions were almost twice higher $(1,15 \%(\mathrm{w} / \mathrm{w})$ and $1,31 \%$ (w/w) respectively) and caffeine content 8-9 times higher $(0,67 \%(\mathrm{w} / \mathrm{w})$ and $0,80 \%(\mathrm{w} / \mathrm{w})$ respectively).

\section{CONCLUSION}

The findings of the present study indicate that Bancha tea is a good source of plant polyphenols which may possess antioxidant, anti-inflammatory and cancer preventive action. The low content of caffeine allows its use by pregnant women, children, and people with cardiovascular diseases and anxiety disorders as a stimulant beverage with proven health properties. All these topics are under investigation and show a high potential for this tea species.

\section{REFERENCES}

1. Balentine DA, Wiseman SA, Bouwens LC. The chemistry of tea flavonoids. Crit Rev Food Sci Nutr. 1997;37(8):693-704.

2. Cabrera C, Artacho R, Gimenez R. Beneficial effects of green tea - a review. J Am Col Nutr. 2006;25:79-99.

3. Chin JM, Merves ML, Goldberger BA, SampsonCone A, Cone EJ. Caffeine Content of Brewed Teas. J Ana Toxicol. 2008:32:702-4.

4. European Pharmacopoeia 6. Edition 2007. Council of Europe (COE) - European Directorate for the Quality of Medicines (EDQM). Strasbourg: Council of Europe, 6.0, 1, 255.

5. Graham HN. Green tea composition, consumption, and polyphenol chemistry. Prev Med. 1992: 21(3):334-50.

6. Iwasaki M, Inoue M, Sasazuki S, Sawada N, Yamaji T, Shimazu T, et al. Green tea drinking and sub- 
sequent risk of breast cancer in a population-based cohort of Japanese women. Breast Cancer Res. 2010;12(5):R88. doi: 10.1186/bcr2756.

7. Najafia NM, Hamidb AS, Afshina RK. Determination of caffeine in black tea leaves by Fourier transform infrared spectrometry using multiple linear regression. Microchem J. 2003;75:151-8.

8. Topalova T, Jelev I, Georgieva S. Quantitative determination of methylxanthines and polyphenols in plant substances. Manage Educat. 2013;9(5):110-4.
9. Yang CS, Lambert JD, Sang S. Antioxidative and anti-carcinogenic activities of tea polyphenols. Arch Toxicol. 2009;83:11-21.

10. Zhelev I, Georgiev K, Georgieva S. Content and pharmacological effects of phenolic compounds in different tea types. Varnenski Medicinski Forum. 2014 (in press), (in Bulgarian). 